Research Paper

\title{
15,16-dihydrotanshinone I inhibits EOMA cells proliferation by interfering in posttranscriptional processing of hypoxia-inducible factor 1
}

\author{
Peiwen Duan ${ }^{1,2}$, Yingying Huang ${ }^{1,3}$, Kai Chen ${ }^{1,2}$, Cheng Cheng ${ }^{1,2}$, Zhixiang $\mathrm{Wu}^{1,2}$ and $\mathrm{Yeming} \mathrm{Wu}^{1,2}$ \\ 1. Department of Pediatric Surgery, Xinhua Hospital, School of Medicine, Shanghai Jiaotong University, Shanghai, China \\ 2. Division of Pediatric Oncology, Shanghai Institute of Pediatric Research, Shanghai, China \\ 3. Department of Reconstructive Surgery, Xinhua Hospital, School of Medicine, Shanghai Jiaotong University, Shanghai, China \\ $\square$ Corresponding authors: Zhixiang Wu, wuzhixiang@xinhuamed.com.cn; Yeming Wu, wuyeming@xinhuamed.com.cn \\ (c) The author(s). This is an open access article distributed under the terms of the Creative Commons Attribution License (https://creativecommons.org/licenses/by/4.0/). \\ See http://ivyspring.com/terms for full terms and conditions.
}

Received: 2021.03.23; Accepted: 2021.06.21; Published: 2021.07.11

\begin{abstract}
Infantile hemangioma $(\mathrm{IH})$, which threatens the physical and mental health of patients, is the most common benign tumor in infants. Previously, we found that 15,16-dihydrotanshinone I (DHTS) was significantly more effective at inhibiting hemangioma proliferation in vitro and in vivo than the first-line treatment propranolol. To investigate the underlying mechanism of DHTS, we used EOMA cells as a model to study the effect of DHTS. We compared the transcriptomes of control and DHTS-treated EOMA cells. In total, 2462 differentially expressed genes were detected between the groups. Kyoto Encyclopedia of Genes and Genomes pathway analysis revealed downregulated activity of the hypoxia-inducible factor 1 alpha (HIF-la) signaling pathway in EOMA cells following treatment with DHTS. Thus, we investigated HIF-1 $\alpha$ expression at protein and mRNA levels. Our results revealed that DHTS downregulated HIF-1 $\alpha$ expression by interfering in its posttranscriptional processing, and the RNA-binding protein HuR participated in this mechanism. Our findings provide a basis for clinical transformation of DHTS and insight into pathogenic mechanisms involved in $\mathrm{IH}$.
\end{abstract}

\section{Introduction}

Infantile hemangioma (IH) is the most common soft-tissue tumor of infancy. The prevalence of $\mathrm{IH}$ in mature neonates is around $4.5 \%[1]$, and it increases with low birthweight and decreasing gestational age, and is as high as $23 \%$ in premature babies with birthweight lower than $1000 \mathrm{~g}$ [2]. Most IH arises within 1-3 weeks after birth and then displays a rapid proliferation period of about 3 months (IH deep in the body of 9-12 months). During this period, approximately $12 \%$ of children have serious complications, for example, IH growing near the eyelids and nose causes permanent visual impairment and disfigurement, while IH growing deep within the body causes respiratory obstruction, nerve compression, gastrointestinal bleeding, and even be life-threatening [3].

Previously, steroids were the first-line treatment for IH. However, a study published in 2008 reported an infant with IH showing signs of improvement after receiving the $\beta$-blocker propranolol [4]. After then, many centers conducted studies and published reports. Propranolol has become the first therapeutic choice for $\mathrm{IH}[5,6]$. However, the side effects of propranolol are inevitable. Sleep disorders, somnolence, and irritability occur in $20 \%-25 \%$ of patients who treated by propranolol. And about $1 \%$ of patients experience side effects such as bronchospasm or bronchiolitis and symptomatic hypotension. In addition, bradycardia, exposure to undiagnosed atrioventricular block, and hypoglycemia are rare but potentially serious side effects [7]. Therefore, it is worthwhile to identify other effective and low-toxic drugs for $\mathrm{IH}$.

15,16-dihydrotanshinone I (DHTS) is a natural 
compound found in Salvia miltiorrhiza. In our previous research, we evaluated the pharmaceutical potential of DHTS on the influence of EOMA cells growth compared with propranolol. We reported that DHTS inhibited the proliferation and angiogenesis of hemangioma more effectively than propranolol [8]. Thus, exploring the mechanism of DHTS on EOMA cells is the focus of our current work.

Studies from other diseases have reported mechanisms of DHTS. For instance, DHTS was verified to exert an anti-inflammatory effect through TLR4/MyD88/NF-kB/MAPK signaling cascades in lipopolysaccharide-stimulated RAW264.7 cells [9]. In addition, DHTS could reverse metabolic reprogramming in colon cancer cells through a PTEN/AKT/HIF-1a-mediated signaling pathway [10]. Moreover, it was reported that DHTS could elicit therapeutic effects against metabolic syndrome. DHTS increases AMPKa phosphorylation and acetyl-CoA carboxylase phosphorylation, thus inhibiting transducer of regulated CREB activity 2 translocation, thereby promoting glucose uptake [11].

An endothelioma cell line (EOMA) originally derived from a spontaneously arising hemangioendothelioma in the $129 / \mathrm{J}$ mouse strain was employed as a well-established model for the study of $\mathrm{IH}$ [12-15]. Its properties could be representative of endothelial cell biology status [16]. In this study, we use EOMA cell line as a model to explore the mechanism of DHTS from the perspective of transcriptomics. Based on the results of bioinformatic analysis, we demonstrated that DHTS regulates HIF-1a expression to inhibit EOMA cells proliferation and angiogenesis and the RNA-binding protein $\mathrm{HuR}$ participates in this process.

\section{Materials and Methods}

\section{Cell Culture and Treatment}

EOMA cell line was purchased from American Type Culture Collection (Manassas, VA, USA) and cultured in 1640 medium (Gibco, Gaithersburg, MD, USA) supplemented with $10 \%$ fetal bovine serum (FBS, Gibco), $100 \mathrm{mg} / \mathrm{L}$ penicillin (Sigma, St. Louis, $\mathrm{MO}, \mathrm{USA}$ ), and $100 \mathrm{mg} / \mathrm{mL}$ streptomycin. Normoxic culture was kept in a humidified incubator with 5\% $\mathrm{CO}_{2}$ and $21 \% \mathrm{O}_{2}$ at $37{ }^{\circ} \mathrm{C}$. The hypoxic culture was kept in a gas-controlled incubator, maintained at $1 \%$ $\mathrm{O}_{2}, 94 \% \mathrm{~N}_{2}$ and $5 \% \mathrm{CO}_{2}$ at $37^{\circ} \mathrm{C}$. Except for hypoxic treatment time, all cells were cultured in normoxic incubator. The powder of DHTS was dissolved in dimethyl sulfoxide (DMSO). Cells of DHTS-group were treated by DHTS solution and cells of control-group were treated by DMSO.

\section{RNA-seq Analysis}

Transcriptomes of EOMA cells were investigated after $24 \mathrm{~h}$ DMSO or DHTS [ $2 \mu \mathrm{M}, \leq$ half maximal inhibitory concentration (IC50)], including three biological replicates per group. Total RNA was extracted from EOMA cells using TRIzol Reagent (15596026, Invitrogen, USA). A total of $3 \mu \mathrm{g}$ of RNA per sample was used as input material for RNA sample preparations. Sequencing libraries were generated using a NEBNext ${ }^{\circledR}$ UltraTM RNA Library Prep Kit (Illumina, San Diego, CA, USA) according to the manufacturer's recommendations and library quality was assessed on an Agilent Bioanalyzer 2100 system (Santa Clara, CA, USA). Sequencing was performed on Illumina HiSeq platform at Beijing Novogene (Beijing, China). Raw reads of fastq format were processed using in-house perl scripts. Hisat2 (v2.04) was used for alignment. Differential expression analysis of three biological replicates per condition was performed using cufflinks/cuffdiff (v2.2.1). Gene Ontology (GO) enrichment analysis of differentially expressed genes was implemented by the clusterProfiler $\mathrm{R}$ package, in which gene-length bias was corrected. GO terms with a corrected P value less than 0.05 were considered significantly enriched by differentially expressed gene (DEG) analysis. The clusterProfiler $R$ package was used to test the statistical enrichment of DEGs in Kyoto Encyclopedia of Genes and Genomes (KEGG) pathway analysis (http://www.genome.jp/kegg/).

\section{Quantitative Reverse Transcription \\ Polymerase Chain Reaction (qRT-PCR)}

Total RNA was isolated with TRIzol reagent. And $1 \mu \mathrm{g}$ of purified RNA from each sample was reverse transcribed to complementary DNA (cDNA). Real-time PCR was performed using a SYBR Green PCR Master Mix kit (Yeason, Shanghai, China), and the following primers: HIF-1a, 5'-GAAACCACCC ATACGTGCTTG-3' (forward) and 5'-AAGTCGTGCT GAATAATACCACT-3' (reverse); GAPDH, 5'-AAGA AGGTGGTGAAGCAGGCATC-3' (forward) and 5'-CGGCATCGAAGGTGGAAGAGTG-3' (reverse).

\section{Stability of mRNA}

EOMA cells were pretreated with DMSO or DHTS for $24 \mathrm{~h}$. After treatment with $2 \mu \mathrm{M}$ actinomycin D (Act D) (A1410, Sigma, USA) to inhibit de novo transcription, RNA of cells was extracted at three time points $(0 \mathrm{~h}, 4 \mathrm{~h}$, and $8 \mathrm{~h})$. Analysis of kinetics for mRNA stability evaluation was carried out by quantitative PCR analysis, after extracting RNA and cDNA synthesis. Residual levels of target mRNA were calculated by comparing to total RNA at $0 \mathrm{~h}$. 


\section{Western Blot}

For preparation of total cell extracts, EOMA cells were washed twice with ice-cold phosphate-buffered saline (PBS) and then lysed in RIPA lysis buffer (BioTime, Alameda, CA, USA). Protein concentration of the extract was determined by Bio-Rad protein assay (Hercules, CA, USA). Next, $20 \mu \mathrm{g}$ protein of each extract was boiled in sodium dodecyl sulfate (SDS) buffer, subjected to SDS-polyacrylamide gel electrophoresis, and then transferred to an immobilon polyvinylidene difluoride membrane (Millipore, Burlington, MA, USA). Membranes were then blocked with $5 \%$ bovine serum albumin in Tris-buffered saline containing Tween. Primary antibodies against $\beta$-Actin, HuR, and HIF-1a (Cell Signaling Technology, Danvers, MA, USA) were used at a concentration of 1 $\mathrm{mg} / \mathrm{mL}$, followed by incubation with an alkaline phosphatase-conjugated goat anti-rabbit secondary antibody (Abcam, Cambridge, UK). Immunofluorescence was detected using a ChemiDoc acquisition instrument (Bio-Rad). ImageJ software was used to convert the image of bands into gray values for statistical analysis.

\section{Immunofluorescence}

Cells were fixed with $4 \%$ formaldehyde for 20 min, permeabilized with $0.3 \%$ Triton X-100 for $10 \mathrm{~min}$, blocked with 5\% bovine serum albumin, and incubated with a primary antibody recognizing HuR (1:50) and HIF-1a (1:50). Secondary antibodies were used to detect primary antibody-antigen complexes with Cy3 (Abcam, Cambridge, UK). Nuclei were stained with 4',6-diamidino-2-phenylindole (DAPI) for $5 \mathrm{~min}$ in a dark room. Finally, images were detected by immunofluorescence microscopy (Leica, Wetzlar, Germany).

\section{RNA immunoprecipitation (RNA-IP)}

To perform cross-linking of HuR and mRNA, cells were fixed in $4 \%$ formaldehyde for $10 \mathrm{~min}$ at room temperature. The reaction was stopped with glycine $(\mathrm{pH} 7,0.25 \mathrm{M})$ for $5 \mathrm{~min}$ at room temperature. Next, cells were washed twice with ice-cold PBS and then resuspended in RIPA Buffer 1 [ $50 \mathrm{mM}$ Tris- $\mathrm{HCl}$ $(\mathrm{pH} 7.5), \quad 1 \%$ Nonidet $\mathrm{P}-40, \quad 0.5 \%$ sodium deoxycholate, $0.05 \%$ SDS, $1 \mathrm{mM}$ EDTA, $150 \mathrm{mM} \mathrm{NaCl}$, and proteinase inhibitors] on the ice for $30 \mathrm{~min}$, and ultrasonication was performed twice for $30 \mathrm{~s}$ each time. The lysate was centrifuged $\left(15 \mathrm{~min}, 4^{\circ} \mathrm{C}, 12000 \times\right.$ g) and the supernatant was extracted. The extract was immunoprecipitated overnight at $4^{\circ} \mathrm{C}$, using protein G-agarose beads preincubated with an anti-HuR or anti-igG antibody. Next, the beads were washed five times with RIPA Buffer 1 and resuspended in RIPA Buffer 2 [50 mM Tris-Cl (pH 7), 5 mM EDTA, 10 mM
DTT, and 1\% SDS]. Cross-linking was reversed by incubation at $70^{\circ} \mathrm{C}$ for $45 \mathrm{~min}$. RNA was purified from immunoprecipitates with TRZol Reagent, treated with RNase-free DNase, and the total RNA was reverse transcribed. The resulting cDNA was subjected to quantitative qRT-PCR using primers against coding regions described in qRT-PCR section.

\section{siRNA Transfection}

siRNA specific for HuR (5'-GGTTGAATCTG CAAAGCTTAT-3') was chemically synthesized by RiboBio (Guangzhou, China). Briefly, EOMA cells were seeded at $1 \times 10^{4}$ cells $/ \mathrm{cm}^{2}$ and transfected with siRNA and INTERFERin (Polyplus-Transfection SA, Illkirch, France) according to the manufacturer's protocol. The cultured medium was replaced with normal 1640 medium with FBS after 6 h. Further administration was performed after transfection for 24-48 h.

\section{Statistical Analysis}

All experiments were repeated three times independently. Data analysis was carried out using SPSS software version 20 (IBM Corporation, Armonk, NY, USA) and GraphPad Prism 5 (GraphPad Software, La Jolla, CA, USA). The results are expressed as mean \pm standard deviation. Significance was determined using a two-tailed paired Student's $t$ test, or one-way analysis of variance (ANOVA) followed by S-N-K (S) or Dunnett's test as appropriate. $p<0.05$ was considered to be statistically significant.

\section{Results}

\section{Transcriptome Profiling of Control and DHTS-Treated EOMA Cells}

Transcriptomes of EOMA cells were investigated according to methods mentioned above. In total, 15573 genes were identified. Firstly, to quality control, we analyzed the correlation of gene expression of biological replicates. $\mathrm{R}^{2}$ was greater than 0.98 , indicating that the correlation of samples was ideal (Figure 1A). Furthermore, Principal component analysis (PCA) was performed to assess transcriptomic relationships among control and DHTS-treated groups based on the combination of PC1 and PC2 (Figure 1B). The largest distinction was $76.46 \%$, between control and DHTS-treated samples (PC1), and the distinction among biological replicates was $7.04 \%$ (PC 2). These results illustrate clear differences between control samples versus DHTS-treated samples. As shown in Figure 1C, hierarchical clustering analysis revealed variations in gene expression between the two groups. To compare gene expression between the two groups, differential 
expression analysis was performed, and significant DEGs were acquired with the criteria of fold change $>$ 1.5 and $P<0.05$. Differential transcriptional analysis revealed differential expression of 2462 genes following treatment with DHTS for $24 \mathrm{~h}$. Among them, 1620 genes were increased and 842 genes were decreased. The volcano plot in Figure 1D shows DEGs between two groups.

To further investigate functional differences in DEGs, GO analysis was employed. GO analysis is divided into three categories: biological process (BP), molecular function (MF), and cellular component (CC). Upon comparing DHTS-treated cells versus control cells, the most significant differences were observed in "metabolic process" (BP), "intracellular" (CC), and "binding" (MF), in addition to other processes (Figure 2A). Among BP category terms, we focused on the process of vascular function, and found that DHTS treatment impacted cardiovascular system development, blood vessel development, blood vessel morphogenesis, and other related processes. As shown in Figure 2B, red bars represent the activated function and blue ones represent the suppressed function. DHTS showed bidirectional regulatory effects on blood vessel development, blood vessel morphogenesis, regulation of vasculature development and so on. While DHTS showed significant down-regulation effects on vasculature development, branching morphogenesis of an epithelial tube, vascular endothelial growth factor receptor signaling pathways and so on.

KEGG pathway analysis identified 127 enriched pathways. Among them, 71 pathways were inhibited (Figure 3A), while 56 were upregulated (Figure 3B). Downregulated and upregulated pathways with the highest enrichment scores were carbon metabolism and p53 signaling, respectively.

B

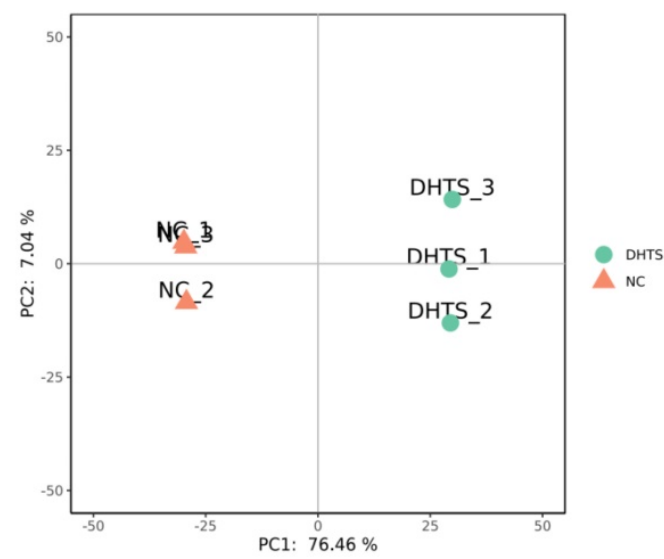

D

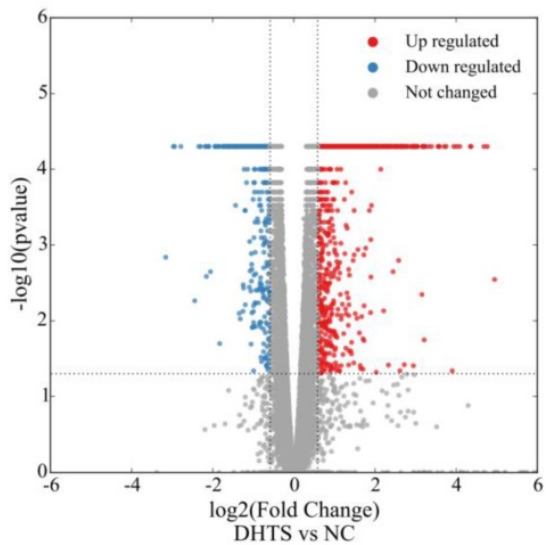

Figure 1. Transcriptome profiling of EOMA cells. NC group: EOMA cells treated by DMSO for $24 \mathrm{~h}$; DHTS group: EOMA cells treated by $2 \mu M$ DHTS for $24 \mathrm{~h}$. (A) Correlation analysis of biological replicates. (B) Principal component analysis. Each group had three biological replicates. (C) Hierarchical clustering of differentially expressed genes between NC group and DHTS group. (D) Volcano plot. X-axis represents the fold change after $\log 2$ conversion, and $Y$-axis represents the fold change after -log 10 conversion. DEGs were acquired with the criteria of fold change $>1.5$ and $\mathrm{P}<0.05$. The red points represent the upregulated genes. The blue points represent the downregulated genes. Gray points represent the non-DEGs. 
A

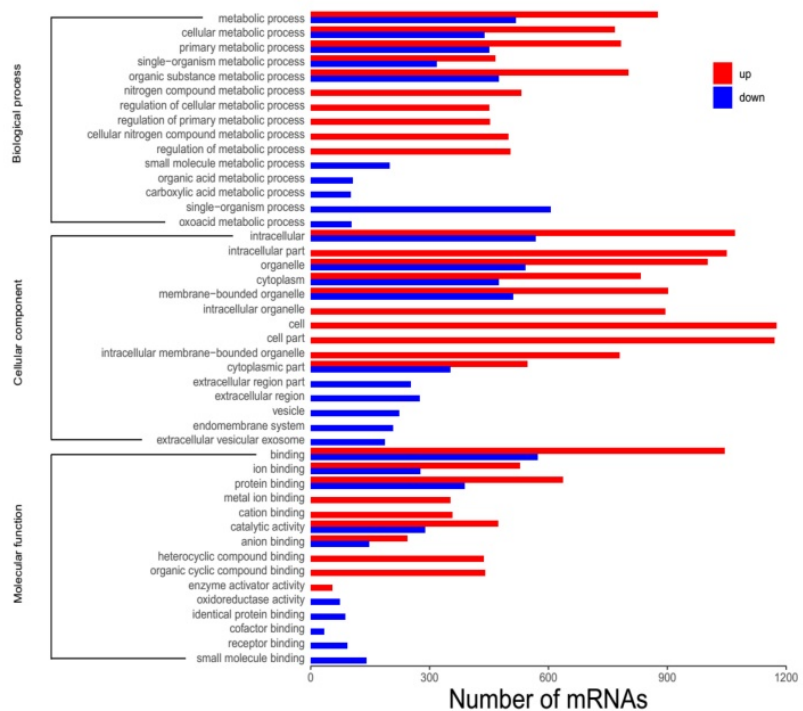

B

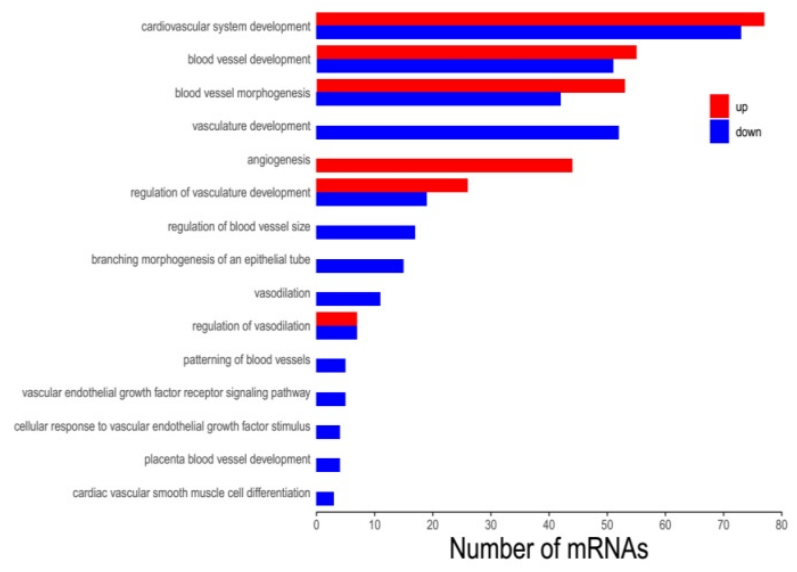

Figure 2. GO analysis of DEGs. (A) GO analysis of DEGs. Bars in red refer to up regulated paths, whereas the blue ones to the down regulated paths. Transcripts were grouped under three main categories: biological processes, cellular components and molecular function, indicated at the left of the figure. The $X$-axis represents the number of genes, and the $\mathrm{Y}$-axis represents the path name. (B) Among BP category terms, the process related to vascular function. Bars in red refer to up regulated paths, whereas the blue ones to the down regulated function.

\section{A}

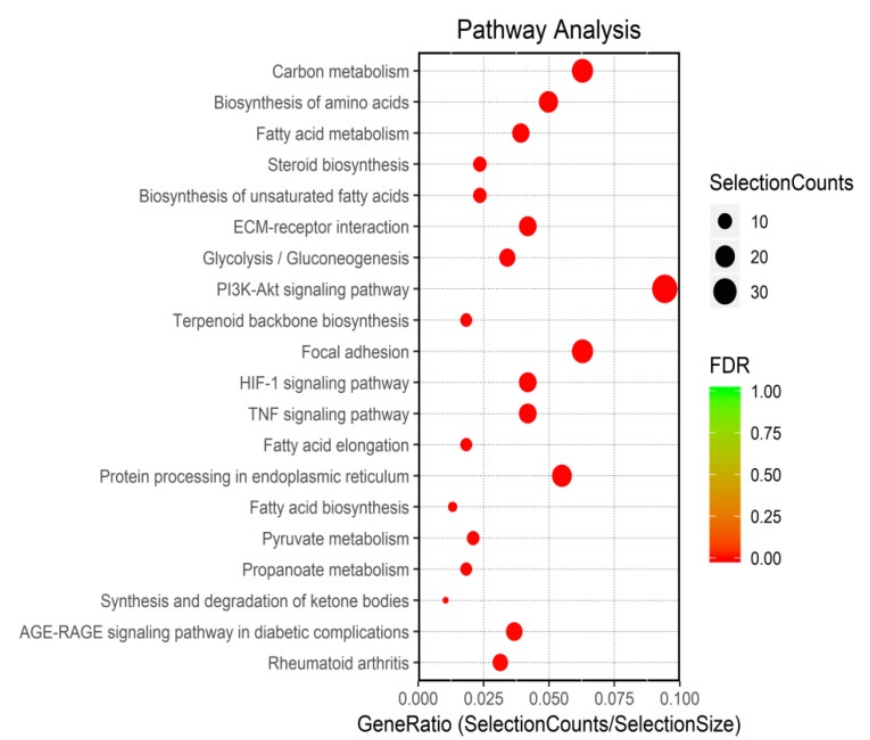

B

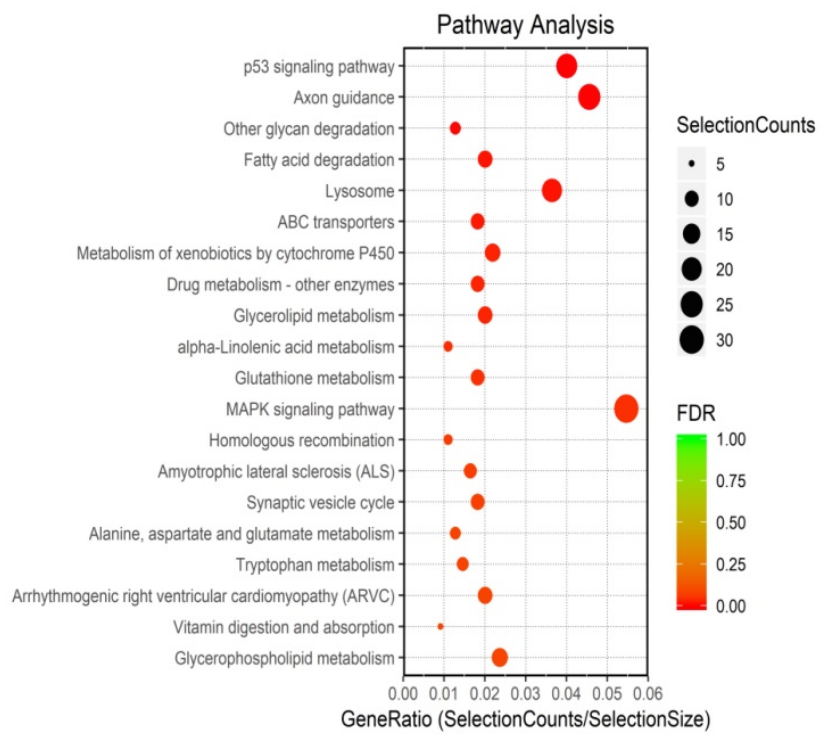

Figure 3. KEGG analysis of DEGs. (A) Top 20 downregulated pathways enrichment distribution of DEGs. The $X$-axis represents gene ratio, and the $Y$-axis represents the path name. The color represents FDR value, and less FDR value means greater intensiveness. The size of the point represents the number of DEGs. (B) Top 20 upregulated pathways enrichment distribution of DEGs. The $X$-axis represents gene ratio, and the $Y$-axis represents the path name. The color represents FDR value, and less FDR value means greater intensiveness. The size of the point represents the number of DEGs.

Then, based on bioinformatics results, we consulted related literatures. We considerd that HIF-1a pathway, one of the top 20 downregulated pathways, is worth to further study. Although the pathogenesis of IH remains unclear, there are several popular hypotheses about it, and hypoxia induction is one of these hypotheses. Much evidence indicated that $\mathrm{IH}$ is induced by intrauterine hypoxia [17]. Coincidentally, the results of RNA-seq revealed significant downregulation of the HIF-1a signaling pathway following DHTS treatment. So, in the following study, we explored whether DHTS regulates HIF-1a and its mechanism. 
A

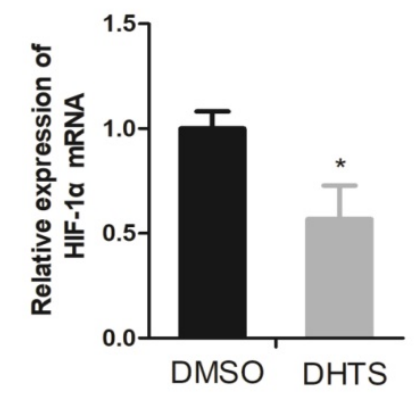

C

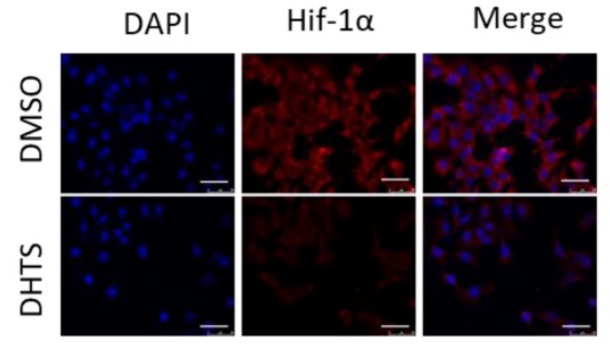

B

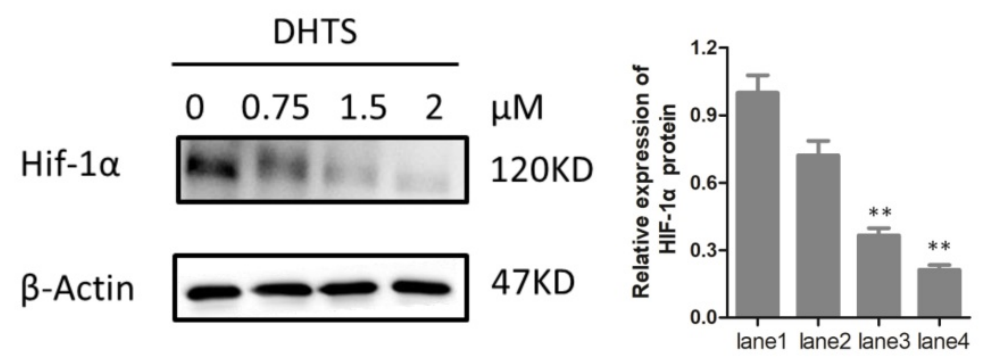

Figure 4. DHTS downregulated HIF-1 $\alpha$ expression at the post-transcriptional level. (A) qRT-PCR reveals that compared with control group, HIF-1 $\alpha$ mRNA in DHTS treated group was significantly decreased. ( $*$ p $<0.05$ ); (B) Western blot analysis. The lanel is the control group. Compared with control group, DHTS could decrease the expression

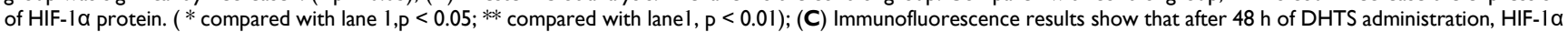
protein expression was reduced compared with control group. Scale bars represent $50 \mu \mathrm{m}$. The magnification was $400 \times$. (** compared with control group, $p<0.01$ ); (D) Stability of HIF-1 $\alpha$ mRNA was detected, the remaining mRNA in control cells was relatively sustained after the addition of Act D within 8h, whereas it was significantly reduced in DHTS-treated cells. (*compared with control group, $\mathrm{p}<0.05$ ).

\section{DHTS Downregulated HIF-1 a Expression at the Post-Transcriptional Level}

To clarify the mechanism by which DHTS acts on HIF-1a signaling pathway, we investigated mRNA and protein levels of HIF-1a. As shown in Figure 4A, after treatment with $2 \mu \mathrm{M}$ DHTS for $24 \mathrm{~h}$, the expression of HIF-1a mRNA was decreased in DHTS-treated group. After treatment with low (0.75 $\mu \mathrm{M})$, medium $(1.5 \mu \mathrm{M})$ and high $(2 \mu \mathrm{M})$ concentrations of DHTS for $48 \mathrm{~h}$, the expression of HIF-1a protein reduced in the DHTS-treated group compared with the control group. Moreover, with the increase of concentration, DHTS was more effective on HIF-1a protein inhibition (Figure 4B). Immunofluorescence results also showed that after 48 $\mathrm{h}$ of $2 \mu \mathrm{M}$ DHTS administration, HIF-1a protein expression was reduced (Figure 4C). We also detected the stability of HIF-1a mRNA by examining the kinetics of the level of remaining HIF-1a mRNA to calculate its half-life. As shown in Figure $4 \mathrm{D}$, the remaining mRNA in control cells was relatively sustained after the addition of Act D, whereas it was significantly reduced in DHTS-treated cells, indicating a significantly shorter half-life.

\section{DHTS Inhibited HuR-Associated HIF-1a Augmentation in Response to Hypoxia}

Since the stability of HIF-1a mRNA and the expression of HIF-1a protein were decreased significantly, we speculated that DHTS may regulate HIF-1a at the post-transcriptional level. It is well known that the ELAVL family is a class of RNA-binding proteins regulating mRNA stability and translation process in response to stimuli such like hypoxia. And post-transcriptional regulation of HIF-1a by HuR (ELAVL1) has already been confirmed in cell models of other diseases [18]. To study the regulation of HIF-1a by HuR in response to hypoxia in EOMA cell model, we detected HIF-1a and HuR protein expression after hypoxic treatment for 0 h, $1 \mathrm{~h}, 2 \mathrm{~h}$, and $3 \mathrm{~h}$, separately. HIF-1a and HuR protein expression was significantly increased 2-3 h after hypoxia (Figure 5A). After hypoxic treatment for $1 \mathrm{~h}$, the expression of HuR protein was statistically higher compared with normoxia group. While for HIF-1a protein, after hypoxic treatment for $2 \mathrm{~h}$, the protein expression between hypoxia group and normoxia group showed statistically different. It may indicate that the overexpression of HIF-1a protein is following after the increase of $\mathrm{HuR}$ protein. Inactive 
A

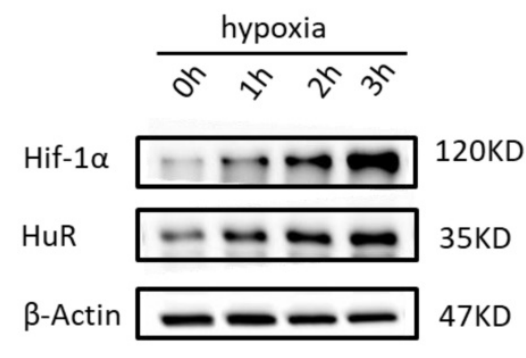

B

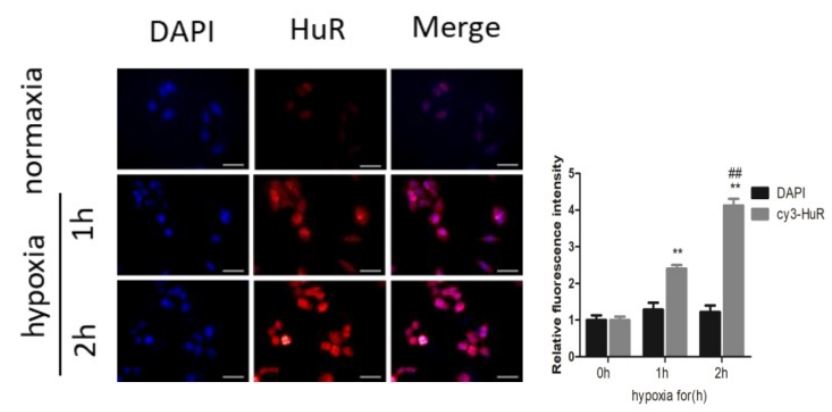

C
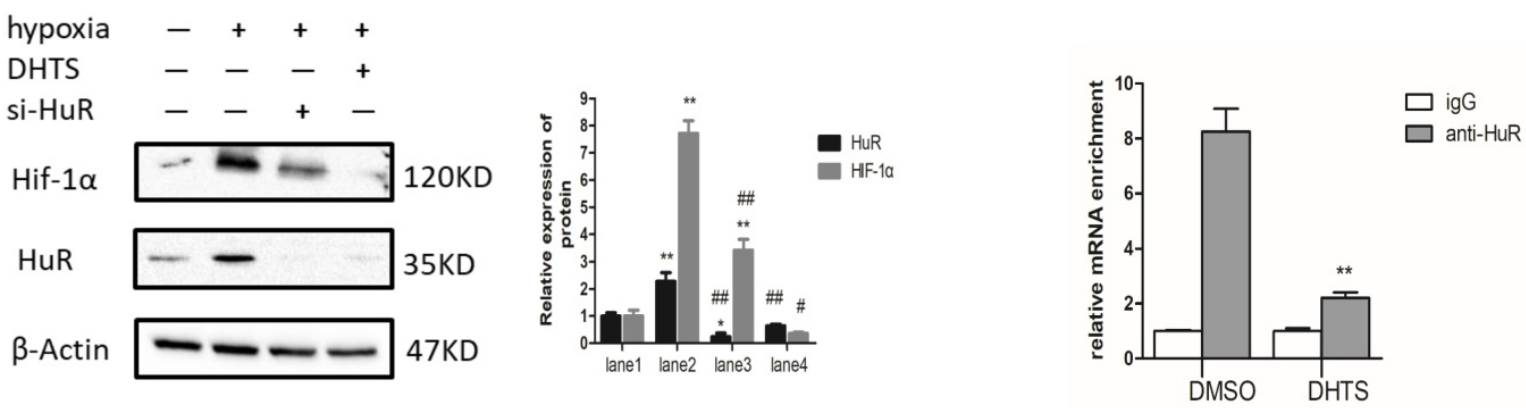

Figure 5. DHTS inhibited HuR-associated HIF-1a augmentation in response to hypoxia. (A) The lanel is the control group. Western blot showed that, compared with the control group, HIF-1 $\alpha$ and HuR expression was significantly increased in response to hypoxia. (*compared with $0 \mathrm{~h}, \mathrm{p}<0.05$; ** compared with $0 \mathrm{~h}$, $\mathrm{p}<0.01$; \#compared with $1 \mathrm{~h}$, $\mathrm{p}<0.05$; \# compared with $1 \mathrm{~h}, \mathrm{p}<0.01$ ); (B) Immunofluorescence results demonstrated augmentation of HuR and distribution to the cytoplasm of HuR in response to hypoxia. Scale bars represent $50 \mu \mathrm{m}$. The magnification was $400 \times$. (** compared with $0 \mathrm{~h}, \mathrm{p}<0.01$; \#\# compared with $1 \mathrm{~h}, \mathrm{p}<0.01)$; (C)The hypoxic treatment maintained for $3 \mathrm{~h}$. The lane1 is the control group. Western blot showed that pretreatment with $2 \mu \mathrm{M}$ DHTS for $24 \mathrm{~h}$ and si-HuR could both inhibit the hypoxia-induced increase in HIF-1 $\alpha$ expression. DHTS could more strongly inhibit HIF-1 $\alpha$ overexpression than si-HuR. (* compared with lane 1, p < 0.05; ** compared with lanel, p < 0.01; \#compared with lane2, p < 0.05; \#\# compared with lane2, $\mathrm{p}<0.01$ ). (D) RNA-IP indicated that HuR could directly bind to HIF-1 $\alpha$ mRNA in EOMA cells, and DHTS interfered with the binding of HuR and HIF-1 $\alpha$ mRNA. ( ** compared with DMSO group, $\mathrm{p}<0.01$ ).

HuR protein mainly expresses in nucleus, and the function of mRNA stability regulation of $\mathrm{HuR}$ depends on its nucleus-plasm shuttle. Therefore, we detected the expression and localization of HuR by immunofluorescence. Immunofluorescence results demonstrated augmentation of $\mathrm{HuR}$ expression within $2 \mathrm{~h}$ of hypoxia. More importantly, HuR was localized in nuclear compartments in normoxia cells, but was apparently distributed to the cytoplasm of hypoxia-exposed cells (Figure 5B).

To further investigate the role of HuR in HIF-1a augmentation in response to hypoxia, we used small interfering RNA (siRNA) to block HuR expression. The results showed that in HuR knocked down cells, the augmentation of HIF-1a protein is significantly inhibited compared with it in negative control ones in response to 3 h-hypoxia (Figure 5C). It indicates that hypoxia-induced overexpression of HIF-1a protein depends on the function of HuR in EOMA cells.

On the other hand, we pretreated EOMA cells with $2 \mu \mathrm{M}$ DHTS for $24 \mathrm{~h}$ before $3 \mathrm{~h}$ hypoxic exposure. The western blot results showed that DHTS could counteract the hypoxia-induced augmentation in HIF-1a expression (Figure 5C).
Moreover, we performed RNA-IP to detect the combination of HuR and HIF-1a mRNA in EOMA cells, as well as the effects of DHTS on this combination. Using an HuR antibody, precipitation levels of HIF-1a mRNA were detected by PCR with reverse transcription. The igG group was used as the negative control to exclude the non-specific binding, and the value of HIF-1a mRNA binding to HuR in each group was showing in Figure 5D. The results indicated that HuR could directly bind to HIF-1a mRNA in EOMA cells, and DHTS interfered with the binding of HuR and HIF-1a mRNA.

\section{Discussion}

IH occurs as a result of abnormal angiogenesis. The pathogenesis of IH has not been completely elucidated. There are several popular hypotheses about its pathogenesis. First of all, hypoxia is considered as an inducer of IH. Under hypoxic environment, as a key transcription factor response to hypoxia stimulation, upregulated HIF-1a could activate pro-angiogenesis mediators such as VEGF and VEGFR [19-21]. Secondly, a theory holds that $\mathrm{IH}$ originated from endothelial progenitor cells, 
which could differentiate into endothelium, adipocytes and pericytes under internal and external stimuli such as tissue ischemia. In addition, the theory of placental origin holds that placental cells may cause embolism during pregnancy and delivery, which is the cause of $\mathrm{IH}$ in embolic tissues of the newborn. [22-23].

In this study, we used EOMA cell line as a model to investigate the mechanism of DHTS on endothelial cells, which represents the possible mechanism of DHTS on IH. We used a transcriptomics approach to evaluate the mechanism by which DHTS inhibits the proliferation of EOMA cells. According to the clues provided by bioinformatic results and the published literatures, we focused on HIF-1a for further research [24]. Epidemiological investigations have shown that the incidence of $\mathrm{IH}$ is closely related to the state of hypoxemia during pregnancy [25]. In low-birthweight premature infants, a group with high incidence of $\mathrm{IH}$, the incidence of intrauterine hypoxia is also three times than that of in normal infants [26]. Analysis of $\mathrm{IH}$ tissue samples revealed abnormally high expression levels of HIF-1a and vascular endothelial growth factor compared with normal tissues [27]. It is well known that HIF-1a is a master transcription factor in the process of angiogenesis through stimulating vascular endothelial growth factor [28]. Moreover, the first-line treatment propranolol, has been demonstrated to exert its suppressive effect on hemangioma through the HIF-1a-VEGF-A angiogenesis axis [29]. Our previous work validated that DHTS could effectively inhibit the proliferation and angiogenesis of EOMA cells [8]. In this study, we revealed DHTS could depress HIF-1a expression levels via a post-transcriptional process.

Under hypoxic conditions, hydroxylation of
HIF-1a protein mediated by von Hippel-Lindau protein is suppressed, which enhances the protein stability of HIF-1a [30]. In addition, the interaction of RNA-binding proteins and HIF-1a mRNA enhances its stability and improves its translation efficiency, thus increasing the total amount of HIF-1a protein [31]. RNA-binding proteins are a class of proteins that can bind and splice target RNA, regulate mRNA stability and translation process, and induce cell differentiation, apoptosis, or proliferation in response to different stimuli and microenvironments [32]. HuR, encoded by the ELAVL1 (embryonic lethal, abnormal vision Drosophila-like 1), is an important RNA-binding protein that plays a major role in hypoxic response mechanisms [33]. Its molecular weight is $36 \mathrm{kD}$. HuR has three RNA recognition motifs, which can selectively combine with adenylate-uridine acid-rich elements in untranslated regions to enhance mRNA stability and carry it to the cytoplasm, thereby promoting translation [34,35]. It has been reported that HuR binds to HIF-1a mRNA to enhance its stability and promote translation efficiency, resulting in an increase in the expression of HIF-1a protein [18]. In our study, we found that in EOMA cells, DHTS could decrease the stability of HIF-1a mRNA as well as the expression of HIF-1a protein. RNA-IP identified the direct binding of $\mathrm{HuR}$ with HIF-1a mRNA in EOMA cells, and the binding was impaired by DHTS. These results suggest that via acting on HuR, DHTS negatively regulates the post-transcriptional process of HIF-1a, thereby inhibiting the proliferation and angiogenesis of EOMA cells. The proposed working model of DHTS on HIF-1a signaling pathway in EOMA cells is shown as Figure 6.

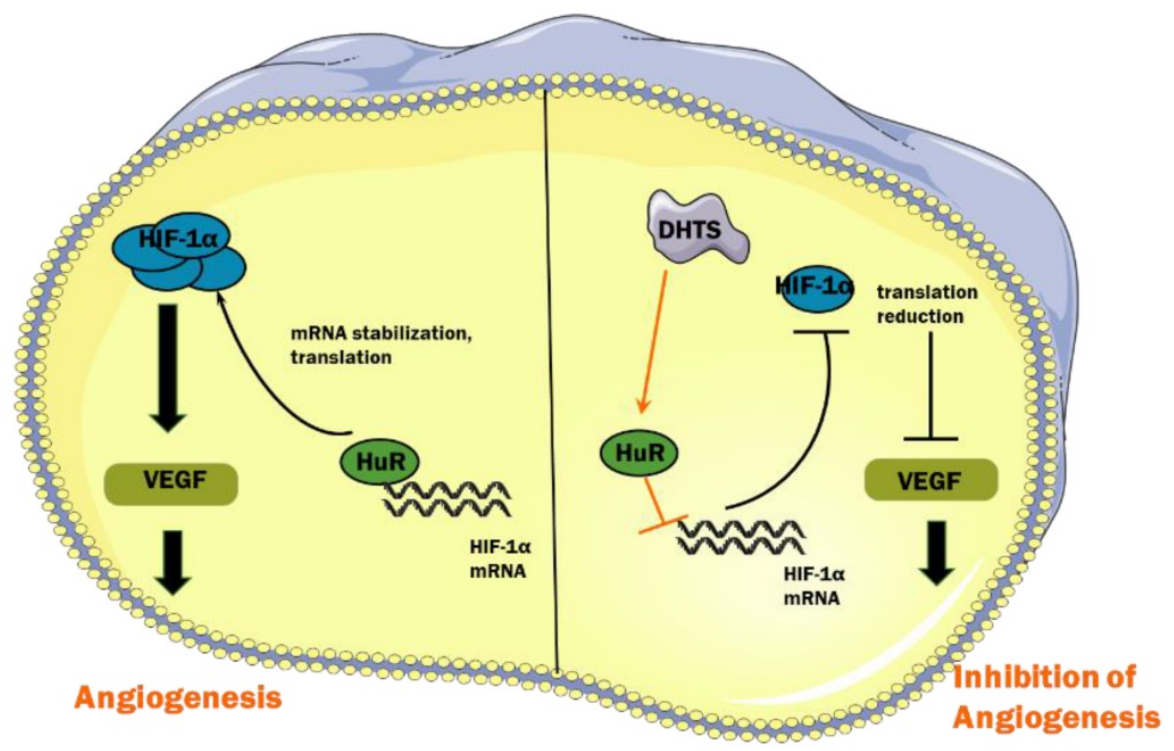

Figure 6. Proposed working model of DHTS on HIF-1 $\alpha$ signaling pathway in EOMA cells. 
It is worth to note that regulating the interaction with HuR may not be the only pathway by which DHTS inhibits HIF-1a protein expression. In figure 5C, compared to HuR-knocked down, DHTS showed stronger effect on inhibiting HIF-1a protein overexpression in response to hypoxia. It has been reported that in human gastric cancer cell model, DHTS could inhibit HIF-1a expression by suppressing its protein accumulation [36]. And DHTS could inhibit HIF-1a protein synthesis through downregulating the activity of mTOR/p70S6K/4E-BP1 and MEK/ERK pathways [37]. These suggests that in EOMA cells, in addition to interfering with the posttranscriptional process of HIF-1a via regulating $\mathrm{HuR}$, other mechanisms of DHTS remain to be explored.

In this study, we proposed for the first time that $\mathrm{HuR}$ participates in the process of EOMA cells proliferation, which indicated that HuR may play a role in the occurrence of hemangioma. Studies have found that $\mathrm{HuR}$ mediates pathological processes such as tumor development, cardiovascular disease, and vascular endothelial abnormalities [38]. For example, in pancreatic ductal adenocarcinoma cells, under hypoxic stress, HuR stabilizes the mRNA of a hypoxia-inducible and pro-oncogenic kinase PIM1, resulting in its protein overexpression, thereby promoting tumor cells growth [39]. It was also reported that after knocked-out HuR, mice exhibited reduced revascularization and tumor angiogenesis, which means that lower expression of HuR could attenuate blood flow and tumor growth [40]. In our study, a siRNA specific to HuR was used to validate its role in inducing HIF-1a overexpression under hypoxia. When HuR was knocked down by siRNA, overexpression of HIF-1a protein was strongly inhibited in response to hypoxia. It suggests that as an important member of RNA-binding proteins, HuR may be worthy of further study in terms of pathogenesis and treatment in hemangioma.

\section{Conclusions}

DHTS could inhibit the proliferation and angiogenesis of EOMA cells through the HIF-1a signaling pathway. Moreover, interfering with the interaction between RNA binding protein $\mathrm{HuR}$ and HIF-1a mRNA may be one of its mechanisms.

\section{Abbreviations}

IH: Infantile hemangioma; DHTS: 15,16-dihydrotanshinone I; HIF-1a: hypoxia-inducible factor 1 alpha; FBS: fetal bovine serum; DMSO: dimethyl sulfoxide; IC50: half maximal inhibitory concentration; GO: Gene Ontology; DEG: differentially expressed gene; KEGG: Kyoto Encyclopedia of Genes and Genomes; PCR:
Polymerase Chain Reaction; Act D: actinomycin D; PBS: phosphate-buffered saline; SDS: sodium dodecyl sulfate; DAPI: 4',6-diamidino-2-phenylindole; RNA-IP: RNA immunoprecipitation; PCA: Principal component analysis; BP: biological process; MF: molecular function; CC: cellular component; siRNA: small interfering RNA; ELAVL1: embryonic lethal, abnormal vision Drosophila-like 1.

\section{Acknowledgments}

We thank all funders listed. We thank Dr. Lin Zeng for the help with bioinformatic analysis.

\section{Funding}

This research was funded by the Natural Science Foundation of China to ZW (No. 81874234), and the Emerging Cutting-edge Technology Joint Research Projects of Shanghai to YW (No.SHDC12018123), and Science and Technology Support Projects in Biomedicine Field of Shanghai Science and Technology Commission to YW (No. 18401931800), and Translational Medicine Collaborative Innovation Project of Shanghai Jiao Tong University School of Medicine to YW (TM201809), and Shanghai Jiao Tong University School of Medicine Doctoral Innovation Fund to PD (No. BXJ201925).

\section{Author Contributions}

Zhixiang $\mathrm{Wu}$ and Yeming $\mathrm{Wu}$ designed the study. Peiwen Duan and Yingying Huang performed the experiments for the study. Kai Chen and Cheng Cheng analysed the data. Peiwen Duan wrote the manuscript. All authors have read and agreed to the published version of the manuscript.

\section{Competing Interests}

The authors have declared that no competing interest exists.

\section{References}

[1] Munden A, Butschek R, Tom W, et al. Prospective study of infantile haemangiomas: incidence, clinical characteristics and association with placental anomalies. Br J Dermatol. 2014; 170:907-913.

[2] Goelz R, Poets C. Incidence and treatment of infantile haemangioma in preterm infants. Arch Dis Child Fetal Neonatal Ed. 2015; 100 : F85-F91.

[3] Haggstrom A, Drolet B, Baselga E, et al. Prospective study of infantile hemangiomas: clinical characteristics predicting complications and treatment. Pediatrics. 2006; 118: 882-887.

[4] Léauté-Labrèze C, Dumas de la Roque E, Hubiche T, et al. Propranolol for severe hemangiomas of infancy. N Engl J Med. 2008; 358: 2649-2651.

[5] Léauté-Labrèze C, Hoeger P, Mazereeuw-Hautier J, et al. A randomized, controlled trial of oral propranolol in infantile hemangioma. N Engl J Med. 2015; 372(8):735-46.

[6] Kim K, Choi T, Choi Y, et al. Comparison of Efficacy and Safety Between Propranolol and Steroid for Infantile Hemangioma: A Randomized Clinical Trial. JAMA Dermatol. 2017; 153:529-536.

[7] Léauté-Labrèze C, Harper J, Hoeger P. Infantile haemangioma. Lancet. 2017; 390: 85-94.

[8] Cai Y, Lv F, Kaldybayeva N, et al. 15, 16-Dihydrotanshinone I Inhibits Hemangiomas through Inducing Pro-apoptotic and Anti-angiogenic Mechanismsin Vitro and in Vivo. Front Pharmacol. 2018; 9:25. 
[9] Yuan R, Huang L, Du L, et al. Dihydrotanshinone exhibits an anti-inflammatory effect in vitro and in vivo through blocking TLR4 dimerization. Pharmacol Res. 2019; 142 :102-114.

[10] Wang L, Yu Z, Ren S, et al. Metabolic reprogramming in colon cancer reversed by DHTS through regulating PTEN/AKT/HIF1a mediated signal pathway. Biochim Biophys Acta Gen Subj. 2018; 1862:2281-2292.

[11] Liu Q, Zhang Y, Lin Z, et al. Danshen extract 15,16-dihydrotanshinone I functions as a potential modulator against metabolic syndrome through multi-target pathways. J Steroid Biochem Mol Biol. 2010; 120:155-63.

[12] Hoak J, Warner E, Chen H, et al. Hemangioma with thrombocytopenia and microangiopathic anemia (Kasabach-Merritt syndrome): an animal model. J Lab Clin Med. 1971; 77:941-950.

[13] Atalay M, Gordillo G, Roy S, et al. Anti-angiogenic property of edible berry in a model of hemangioma. FEBS Lett. 2003; 544(1-3):252-7.

[14] Ou J, Lian W, Qiu M, et al. Knockdown of IGF2R suppresses proliferation and induces apoptosis in hemangioma cells in vitro and in vivo. Int J Oncol. 2014; 45(3):1241-9.

[15] Zeng L, Tao C, Liu Z, et al. Preparation and Evaluation of Cubic Nanoparticles for Improved Transdermal Delivery of Propranolol Hydrochloride. AAPS PharmSciTech. 2020; 21(7):266.

[16] Obeso J, Weber J, Auerbach R. A hemangioendothelioma-derived cell line: its use as a model for the study of endothelial cell biology. Lab Invest. 1990; 63(2):259-69.

[17] Drolet BA, Frieden I. Characteristics of infantile hemangiomas as clues to pathogenesis: does hypoxia connect the dots? Arch Dermatol. 2010; 146:1295-1299.

[18] Galbán S, Kuwano Y, Pullmann R, et al. RNA-binding proteins HuR and PTB promote the translation of hypoxia-inducible factor 1alpha. Mol Cell Biol. 2008; 28:93-107.

[19] Yin RR, Hao D, Chen P. Expression and correlation of MMP-9, VEGF, and p16 in infantile hemangioma. EurRev Med Pharmacol Sci. 2018; 22:4806-11.

[20] Oszajca K, Szemraj J, Wyrzykowski D, et al. Single-nucleotide polymorphisms of VEGF-A and VEGFR-2 genes and risk of infantile hemangioma. Int J Dermatol. 2018; 57(10):1201-1207.

[21] Ji Y, Chen S, Li K, et al. Signaling pathways in the development of infantile hemangioma. J Hematol Oncol. 2014; 7:13.

[22] Darrow DH, Greene AK, Mancini AJ, et al. Diagnosis and Management of Infantile Hemangioma. Pediatrics. 2015; 136(4):e1060-104.

[23] Janmohamed SR, Madern GC, de Laat PC, et al. Educational paper: Pathogenesis of infantile haemangioma, an update 2014 (part I). Eur J Pediatr. 2015; 174(1):97-103.

[24] Léauté-Labrèze C, Prey S, Ezzedine K. Infantile haemangioma: part I. Pathophysiology, epidemiology, clinical features, life cycle and associated structural abnormalities. J Eur Acad Dermatol Venereol. 2011; 25:1245-1253.

[25] Drolet B, Swanson E, Frieden I, et al. Infantile hemangiomas: an emerging health issue linked to an increased rate of low birth weight infants. J Pediatr. 2008; 153:712-715.

[26] Colonna V, Resta L, Napoli A, et al. Placental hypoxia and neonatal haemangioma: clinical and histological observations. Br J Dermatol. 2010; 162:208-209.

[27] Kleinman M, Greives M, Churgin S, et al. Hypoxia-induced mediators of stem/progenitor cell trafficking are increased in children with hemangioma. Arterioscler Thromb Vasc Biol. 2007; 27:2664-2670.

[28] Zimna A, Kurpisz M. Hypoxia-inducible factor-1 in physiological and pathophysiological angiogenesis: applications and therapies. Biomed Res Int. $2015 ; 2015: 549412$.

[29] Chim H, Armijo BS, Miller E, et al. Propranolol induces regression of hemangioma cells through HIF-1a-mediated inhibition of VEGF-A. Ann Surg. 2012; 256(1):146-56.

[30] Koyasu S, Kobayashi M, Goto Y, et al. Regulatory mechanisms of hypoxia-inducible factor 1 activity: Two decades of knowledge. Cancer Sci. 2018;109: 60-571.

[31] Rossignol F, de Laplanche E, Mounier R, et al. Natural antisense transcripts of HIF-1alpha are conserved in rodents. Gene. 2004; 339:121-130.

[32] Doller A, Akool el-S, Huwiler A, et al. Posttranslational modification of the AU-rich element binding protein $\mathrm{HuR}$ by protein kinase Cdelta elicits angiotensin II-induced stabilization and nuclear export of cyclooxygenase 2 mRNA. Mol Cell Biol. 2008; 28:2608-2625.

[33] Gallouzi I, Steitz J. Delineation of mRNA export pathways by the use of cell-permeable peptides. Science. 2001;294 :1895-1901.

[34] Mukherjee N, Corcoran D, Nusbaum J, et al. Integrative regulatory mapping indicates that the RNA-binding protein HuR couples pre-mRNA processing and mRNA stability. Mol Cell. 2011; 43:327-339

[35] Lebedeva S, Jens M, Theil K, et al. Transcriptome-wide analysis of regulatory interactions of the RNA-binding protein HuR. Mol Cell. 2011; 43:340-352.

[36] Dat N, Jin X, Lee J, et al. Abietane diterpenes from Salvia miltiorrhiza inhibit the activation of hypoxia-inducible factor-1. J Nat Prod. 2007; 70(7):1093-7.

[37] Li J, Mi C, Ma J, et al. Dihydrotanshinone I inhibits the translational expression of hypoxia-inducible factor-1a. Chem Biol Interact. 2015; 240:48-58.

[38] Rhee W, Ni C, Zheng Z, et al. HuR regulates the expression of stress-sensitive genes and mediates inflammatory response in human umbilical vein endothelial cells. Proc Natl Acad Sci U S A. 2010; 107: 6858-6863.

[39] Blanco F, Jimbo M, Wulfkuhle J, et al. The mRNA-binding protein HuR promotes hypoxia-induced chemoresistance through posttranscriptional regulation of the proto-oncogene PIM1 in pancreatic cancer cells. Oncogene. 2016; 35:2529-41.

[40] Chang S, Elemento O, Zhang J, et al. ELAVL1 regulates alternative splicing of eIF4E transporter to promote postnatal angiogenesis. Proc Natl Acad Sci U S A. $2014 ; 111: 18309-14$. 